\title{
Neue Medien: Berufliche Chancen für Frauen?
}

\author{
Thomas Döbler / Birgit Stark
}

Mit dem Wandel zur Informationsgesellschaft wird oftmals eine bessere Vereinbarkeit von Arbeits- und Lebenswelt prognostiziert. Fest gefügte Zuständigkeiten scheinen sich $z u$ verändern, wobei insbesondere Frauen von den weit reichenden Veränderungen profitieren sollen: Die Flexibilisierung der Erwerbsarbeit in den Dimensionen Menge, Zeit und Ort kann für die Realisierung von individuellen Lebensentwürfen nutzbar gemacht werden. Darüber hinaus verändern sich Arbeitsanforderungen und -inhalte. Neben Fachwissen werden auch zunehmend Sozial- und Methodenkompetenzen wie Teamund Kommunikationsfähigkeit oder Problemlösefähigkeit nachgefragt, Fähigkeiten, die traditionell als, weiblich' definiert und damit Frauen zugeordnet werden. Eine Analyse auf Basis von derzeit verfügbaren Daten der gegenwärtigen arbeitsmarktbezogenen Entwicklungen im Bereich der Neuen Medien (Ausbildungs-, Beschäftigungsverhältnisse etc.) deutet aber eher darauf hin, dass auch hier die traditionelle Geschlechtersegregation reproduziert wird. Es bleibt abzuwarten, ob und inwieweit es den Frauen gelingen wird, die sich zweifelsohne ergebenden Optionen noch besser auszuschöpfen.

Keywords: Neue Medien, Frauen, Beschäftigungsoptionen, Flexibilisierungstendenzen, Qualifikationsanforderungen

\section{Ausgangsüberlegungen}

Entstehung und Diffusion neuer Dienstleistungen, insbesondere im Bereich der Informations- und Kommunikationswirtschaft, bilden ein Kernstück des wirtschaftlichen Strukturwandels, der im öffentlichen Diskurs als Herausbildung einer „New Economy“ bezeichnet wird. In einer kurzen euphorisierten Phase wurde diese New Economy und mit ihr vor allem die „Neuen Medien“ als neuer Wirtschaftsbereich gehandelt, in dem die Gesetze der alten Ökonomie außer Kraft gesetzt sein sollten. Auch wenn die Illusion „immer währenden“ Wachstums nur kurz währte, werden mit diesem Bereich nach wie vor „neue“ Arbeitsmodelle und -strukturen assoziiert: Basierend auf IuK-Technologien und neuen Formen der Wertschöpfung (wissensbasiert) - so die Argumentation - gewinnen unternehmensinterne und -externe Netzwerkstrukturen als Organisationsprinzip an Bedeutung, während gleichzeitig damit die traditionell hierarchischen Strukturen an Gewicht verlieren, wenn nicht überwunden werden; eigenständig, zeitlich und inhaltlich flexibel werde das Arbeiten nun in wechselnden Teams organisiert. Ein derartiges „Klima“ des Arbeitens begünstige nun auch das Überwinden beruflich verfasster Geschlechtersegregationen und der traditionell tätigkeitsbezogenen Geschlechterhierarchie. Und da die in diesem Bereich vermehrt geforderten Qualifikationen, die so genannten „social skills“, die Team- und flexiblen Kooperationsfähigkeiten, die kommunikativen Kompetenzen, ja gerade Frauen für und in diesen Bereichen prädestinierten, wird die New Economy im Allgemeinen und die Neuen Medien im Speziellen als große Chance für die Frauen postuliert. Mit der Entwicklung zur „Kommunikationsgesellschaft" - so die Kurzformel - werden sich dabei nicht nur rein quantitativ wachsende Tätigkeitsoptionen ergeben, sondern diese schaffe auch den Durchbruch zu qualifizierten und Führungspositionen für Frauen und trage damit bei, geschlechtsspezifische Benachteiligungen und Diskriminierungen in der Arbeitswelt zu mindern. Ob das dyna- 
mische Wachstum der Neuen Medien mit fraglos vielfältigen Impulsen auf Beschäftigung und Arbeitspolitik auch Emanzipationspotenziale gleichsam per se mit sich führt, muss sich jedoch erst noch empirisch bestätigen. Auch früher schon wurden immer wieder im Zusammenhang mit bestimmten Technologieschüben oder globalen Strukturverschiebungen, etwa dem sektoralen Wandel von der Industrie- zur Dienstleistungsgesellschaft, nachhaltige und grundlegende Verbesserungen hinsichtlich der Erwerbs- und Berufstätigkeit von Frauen prognostiziert - bei einer ex post Betrachtung jedoch meist überschätzt. Ein Blick auf die Entwicklung der weiblichen Erwerbstätigkeit und die Beschäftigtensituation von Frauen, innerhalb und außerhalb der Medien, mag vielleicht helfen, die insgesamt noch unter großem Datenmangel leidende Diskussion zu den beruflichen Chancen - Risiken scheint es kaum zu geben - von Frauen in den Neuen Medien etwas „nüchterner“ gestalten zu können.

Vor einem knapp zu haltenden Streifzug durch die jüngere Entwicklung der weiblichen Beschäftigung in Deutschland ist zunächst jedoch noch das Objekt der großen Hoffnungen, also die „Neuen Medien“ selbst etwas zu präzisieren. Denn weder der Ausdruck noch der Bereich „Neue Medien“ kann analytisch sinnvoll und eindeutig abgegrenzt werden: dauernd werden neue Medien fortentwickelt. Das, was neu ist, ist immer nur neu relativ zu einem alten Medium. Es gab immer neue und alte Medien.

Was in der gegenwärtigen Diskussion um Neue Medien Definitionen noch erschwert und Abgrenzungen unscharf werden lässt, ist die partielle Verschmelzung und Überlagerung vormals klar separierter Wirtschaftsbranchen und -bereiche, vor allem der Telekommunikations- und der Computerindustrie, mit dem Medienbereich. Insofern finden sich auch stark variierende Auffassungen von Inhalt und Bedeutung der Neuen Medien und auch die Unterscheidung zu konkurrierenden Begriffen wie etwa Multimedia oder IT-Bereich bleibt vielfach vage. Diese begriffliche und inhaltliche Uneindeutigkeit erschwert nun insbesondere einen bereichsspezifischen Zugang zu den Neuen Medien oder genauer: zur Neuen Medienbranche. So wird etwa Multimedia heute als Oberbegriff für eine Vielzahl neuer bzw. neuartiger Produkte und Dienstleistungen aus dem Computer-, Telekommunikations- und Medienbereich verwendet, doch Multimediaberufe und -tätigkeiten finden sich keineswegs, vielleicht sogar nur zum kleineren Teil, nur in der Medienbranche, sondern eben auch im Handel, bei Banken oder in der Weiterbildung. Ähnliches gilt auch für IT-Berufe und -Tätigkeiten.

Auch wenn niemand ernsthaft behaupten wird, dass Unternehmen dieser „alten“ Branchen selbst durch die vermehrte Implementierung von Multimedia-Arbeitsplätzen, von Arbeitsplätzen, die sich der Neuen Medien direkt und umfassend bedienen, zu (Neuen) Medienunternehmen mutieren, so schwierig gestaltet sich doch schon die rein zahlenmäßige Erfassung und Zuordnung von Beschäftigten auf die Neue Medienbranche, die Telekommunikations- und Computerindustrie und auf Medienarbeitsplätze in den traditionellen Branchen. Denn sowohl die Statistiken zu Ausbildungen und Studiengängen als auch zur Beschäftigung differenzieren im Bereich der Neuen Medienberufe kaum nach tätigkeits- und branchenspezifischem Einsatz. Hinzu kommt, dass die durch die Dynamik der Neuen Medien ausgelöste „Goldgräberstimmung“ in den zurückliegenden Jahren sehr viel Raum für Quereinstiege geboten hat, eine Entwicklung, die mit der ersten Krise in diesem Bereich jedoch rasch zu einem Ende gekommen zu sein scheint. Darüber hinaus ist auch in den Neuen Medien nicht alles neu; klassische unternehmerische Funktionen, z. B. Personal, Marketing oder Controlling, sind auch in den Neuen Medien relevant und werden, spätestens nach den jüngsten Turbulenzen, nicht viel anders als in einem alten Medienunternehmen betrieben und organisiert wer- 
den - also auch mit ganz traditionellen Berufsbildern, ja u. U. sogar Berufskarrieren in diesen Funktionsbereichen.

Eine Diskussion potenzieller geschlechtsemanzipatorischer Bedingungen und Strukturen in der Neuen Medienbranche hat sowohl diese funktionalen als auch bereichsspezifischen Zuordnungsschwierigkeiten, diese mehrfachen Überlagerungen von „Alt" und „Neu“ als auch die defizitäre Datenlage stets zu bedenken, Schlussfolgerungen und Thesen weisen unvermeidlich zu einem Teil spekulativen Charakter auf. Ausgehend von allgemeinen Bedingungen und Entwicklungen weiblicher Erwerbstätigkeit werden wir jedoch versuchen, die beruflichen Perspektiven geschlechtsbezogen in den Neuen Medien zu erörtern. Folgen wollen wir dabei einer eher pragmatischen Abgrenzung von Neuen Medien:

Allgemein kann die Medienbranche als ein Bereich definiert werden, in dem Anbieter (Unternehmen) Informationen über Sachverhalte und Ereignisse der Politik, Wirtschaft, Wissenschaft, Kultur, Unterhaltung etc. sowie künstlerische Bild- und Tonwerke produzieren und mittels geeigneter Vertriebswege und Übertragungstechnologien verbreiten. Konkret werden damit die klassischen Medienunternehmen bezeichnet, also Printmedien (Zeitungs-, Zeitschriften-, Buchverlage) und Rundfunkunternehmen, aber auch die so genannten Neuen Medienunternehmen - hierunter fallen neben den „älteren“ Neuen Medienunternehmen, wie z. B. Film- und Tonträgerherstellern, vor allem in jüngster Zeit das Internet. Als Unternehmen der Neuen Medienbranche sollen im Folgenden nun die Unternehmen definiert werden, die technisch, journalistisch oder künstlerisch, kaufmännisch oder beratend, in der Produktion oder dem Vertrieb der Medien „CD“, „DVD“, „Internet“ aktiv sind. Ein solchermaßen eingegrenztes Verständnis der Neuen Medienbranche umfasst damit

- Tätigkeiten der technischen Produktion von Medienprodukten (Webdesign, Systemadministration, Softwareentwicklung),

- Tätigkeiten, die direkt auf die Inhalte der Neuen Medienproduktion Einfluss nehmen (Redaktion, Journalisten/innen, Lektoren/innen etc.),

- Tätigkeiten, die in spezifischen Funktionsbereichen die jeweiligen Neuen Medienbetriebe nach außen oder nach innen repräsentieren (z. B. Führungskräfte oder Existenzgründer/innen) sowie

- Tätigkeiten, die Beratungsdienstleistungen für Multimedia-Auftritte/-Konzepte beinhalten (Mediaberatung).

\section{Eckdaten zur Beschäftigung und zur Qualifikation von Frauen}

Nahezu unabhängig von der konjunkturellen und strukturellen Entwicklung, d.h. auch unabhängig von der unterschiedlichen Arbeitsmarktsituation, nahm die Zahl der Frauen in Westdeutschland, die berufstätig sind und sein wollen, seit Mitte der 60er Jahre bis heute stetig zu. Insbesondere die stärkere Erwerbsorientierung von verheirateten Frauen in mittleren Altersgruppen (zwischen 35 und 50 Jahren) ist für die gestiegene weibliche Erwerbsquote ${ }^{1}$, die mittlerweile über $60 \%$ liegt, verantwortlich: Frauen unterbrechen ihre Erwerbstätigkeit wegen Familienpflichten seltener und im Falle einer Unterbrechung sind die Unterbrechungszeiten inzwischen wesentlich kürzer geworden. Dies

1 Die Erwerbsquote errechnet sich aus dem Anteil der Erwerbspersonen (Erwerbstätige und Erwerbslose) an der Wohnbevölkerung im erwerbsfähigen Alter (15 bis unter 65 Jahre) (BundLänder-Kommission, 2000: 24). 
hängt u.a. maßgeblich vom steigenden Schul- und Berufsbildungsniveau der Frauen, nicht zuletzt aber auch von der Verfügbarkeit von Teilzeitstellen ab (Bundesanstalt für Arbeit, 2000: 388). Hinzu kommt, dass für den überwiegenden Teil der Frauen Berufstätigkeit heute einen eigenständigen Wert aufweist, der über rein ökonomische Aspekte hinausgeht und bei dem vor allem intrinsische Motive überwiegen (Engelbrech/Reinberg, 1998: $53 \mathrm{ff}.)^{2}$

Frauen sind zum weit überwiegenden Anteil (80\%) im Dienstleistungssektor beschäftigt (Männer zu rund 50\%), dagegen zu nur $18 \%$ im produzierenden Gewerbe tätig (hier liegt der Anteil bei Männern bei 45\%). Beschäftigungsschwerpunkte sind dabei öffentliche und private Dienstleistungen (ohne Öffentliche Verwaltung) sowie Handel und Gastgewerbe. Rund zwei Drittel davon arbeiten in den so genannten primären Dienstleistungen $^{3}$ (Bundesanstalt für Arbeit, 2000: 392f.). Dieser Schwerpunkt auf die Dienstleistungen war, zumindest für die Frauen in den alten Bundesländern, ein wesentlicher Grund dafür, dass sie in den neunziger Jahren von dem insgesamt kräftigen Beschäftigungsrückgang im sekundären Sektor kaum getroffen wurden - die Arbeitslosenquote der Frauen hat sich damit auch der der Männer angeglichen - und sogar noch einen Beschäftigungszuwachs verzeichnen können. Allerdings entstanden die Beschäftigungsgewinne überwiegend auf Grundlage von Teilzeitbeschäftigungen (Engelbrech/Jungkunst, 1999: 22). Mittlerweile sind in den alten Bundesländern ca. 40\% der erwerbstätigen Frauen (Männer zu 5\%) teilzeitbeschäftigt (in den neuen Ländern, wo Vollzeitarbeit nach wie vor einen höheren Stellenwert genießt, ${ }^{4}$ sind es $22 \%$ ).

Als entscheidender Wettbewerbsfaktor auf dem Arbeitsmarkt über alle Konjunkturzyklen hinweg zeigt sich die Qualifikation. Ohne abgeschlossene Ausbildung wächst das „Beschäftigungsrisiko“; während Un- und Geringqualifizierte eine deutliche Abnahme in der Beschäftigung verzeichnen, gibt es z. B. bei den akademischen Berufen Beschäftigungsgewinne.

Das formale Bildungsniveau von Mädchen und Frauen ist in den letzten Jahrzehnten deutlich gestiegen und liegt mittlerweile über dem der Männer, dennoch zeigt sich eine bemerkenswerte Konstanz tradierter Verhaltensmuster und Einstellungen sowohl in der geschlechtsspezifischen Wahl von Fächern und Fachkombinationen an Schulen und Hochschulen als auch von beruflichen Ausbildungen, womit die Erwerbsbiografien und beruflichen Karrieren nachhaltig beeinflusst werden: So wählen Schülerinnen nach wie vor z. B. deutlich häufiger als Schüler Sprachen, Musik und Kunst, dagegen deutlich weniger Mathematik oder Physik als Leistungsfach (Bund-Länder-Kommission, 2000: 29). Auch bei der Wabl der Studienfächer bleiben, ungeachtet der fast erreichten Parität in den Wirtschaftswissenschaften, geschlechtsspezifische Präferenzen hartnäckig erhalten: Auf der einen Seite ein starkes weibliches Übergewicht in Studiengängen für das Lehramt, der Sprach- und Kulturwissenschaften und des Sports, auf der anderen Seite ein entsprechendes Untergewicht in den ingenieurwissenschaftlichen Studiengängen, inkl. In-

2 In den neuen Bundesländern ist die weibliche Erwerbsquote zwar im Vergleich zu DDR-Zeiten deutlich gesunken, liegt jedoch weiterhin über der der alten Bundesländer (vgl. ausführlicher ANBA; 2000: 388).

3 Hierunter fallen Ein- und Verkaufen, Kassieren, allgemeine Bürotätigkeiten, Lager- und Versand, Reinigen oder Bewirten.

4 So geben z. B. über die Hälfte der teilzeitbeschäftigten Frauen in den neuen Ländern gemäß Mikrozensus an, dass sie nur deshalb einen Teilzeitarbeitsplatz haben, weil Vollzeitarbeitsplätze fehlen. 
formatik: Hier liegen die Frauenanteile trotz gewisser Steigerungsraten bei unter 20\% (Bund-Länder-Kommission, 2000: 44).

Ein noch stärker geschlechtsspezifisches Verhalten zeigt sich bei der Wabl des Ausbildungsberufes. In den 25 am häufigsten gewählten Berufen (von rund 400 möglichen) bei Frauen werden 79\% aller Neuabschlüsse getätigt - bei Männern beträgt dieser Anteil 60\% (Bundesinstitut für Berufsbildung, 2001). Weniger diese Konzentration auf nur wenige, als vielmehr vor allem auf „typisch weibliche“ Ausbildungsberufe mit meist eingeschränkten Entwicklungs- und Aufstiegsmöglichkeiten sowie geringeren Durchschnittsverdiensten muss dabei als ein entscheidendes Starthandicap von Frauen für ihre gesamte Erwerbsbiografie gewertet werden.

Auch die Nachfrage nach den zehn neuen Ausbildungsberufen ${ }^{5}$, mit denen das duale System der Berufsausbildung 1997 auf die Veränderungen der Medien- und Informationswirtschaft reagierte, lässt Geschlechtsspezifika erkennen: Einzig in der grafischen Industrie (Druck, Vorstufe, Verlage, Agenturen), die durch die Digitalisierung in besonderem Maße von Veränderungsprozessen und Reorganisationen betroffen ist, zeigt sich bei den Ausbildungszahlen in den hier neu entstandenen Berufen (Fotomedienlaborant/in, Mediengestalter/in für Digital- und Printmedien, Werbe- und Medienvorlagenhersteller/in) ein geschlechtsspezifisch sehr ausgewogenes Bild: Jeweils etwas über 50\% der neu abgeschlossenen Ausbildungsverträge - beim Berufsbild der Fotomedienlaborantin sogar über $60 \%$ - sind 1998 von Frauen getätigt worden. Auch wenn sich in bestimmten Fachrichtungen dieser neuen Berufe ${ }^{6}$ wiederum bestätigt, dass in den kreativen und gestaltungsorientierten Bereichen mehr, in den technisch orientierten weniger Mädchen ausgebildet werden, zeigen sich in moderneren Unternehmen doch bereits Auflösungserscheinungen hinsichtlich dieser Strukturen (Klatt/Richter-Witzgall, 2000: $33 \mathrm{ff}$. .). Im audiovisuellen Bereich (Film-, Fernseh- und Hörfunkproduktion), in dem nur in vergleichsweise geringem Umfang ausgebildet wird, sind die Arbeitsbedingungen ohnehin familien- und damit tendenziell frauenfeindlich. Die Ausbildungszahlen in den hier neu entstandenen Berufen (Kaufmann/-frau für audiovisuelle Medien, Mediengestalter/in in Bild und Ton, Film- und Videoeditor/in) zeigen bei den Berufen des/der Kaufmanns/-frau ein positives (63\%), beim Beruf Mediengestalter/in ein negatives Bild für Frauen (30\%). Die Gesamtbedingungen der Branche lassen allerdings vermuten, dass weniger die Ausbildung an sich, sondern die fehlenden Ausbildungsstandards sowie insbesondere die extremen Arbeitsformen ein Hauptproblem für eine stärkere Partizipation der Frauen sein dürften (Klatt/Richter-Witzgall, 2000: 38ff.). In den vier im engeren Sinne für den Multimediabereich geschaffenen Ausbildungsberufen (Fachangestellte/r für Medien- und Informationsdienste, Informatikkaufmann/frau, Fachinformatiker/in, I- und TK-Kaufmann/frau, I- und TK-Elektroniker/in) sind Frauen deutlich unterrepräsentiert. Offensichtlich sind dabei selbst- und fremdselektive Mechanismen nicht nur in den techniklastigen Berufen unverändert wirksam, sondern dehnen

5 Das sind Fotomedienlaborant/in, Fachinformatiker/in, Informations- und Telekommunikationssystem-Elektroniker/in, Informations- und Telekommunikationssystem-Kaufmann/frau, Informatikkaufmann/frau, Fachangestellte/r für Medien- und Informationsdienste, Film- und Videoeditor/in, Mediengestalter/in Bild und Ton, Kaufmann/frau für audiovisuelle Medien und Mediengestalter/in für Digital- und Printmedien.

6 So besteht z. B. das neue Berufsbild des/der Mediengestalters/in für Digital- und Printmedien aus den folgenden vier Fachrichtungen: Mediendesign, Medienoperating, Medientechnik und Medienberatung. 
sich auch auf die kaufmännischen Berufe aus, die ansonsten mit 62\% eine Domäne der Frauen darstellen.

Tabelle 1: Entwicklung der IT-Berufe

\begin{tabular}{lcccccc}
\hline $\begin{array}{l}\text { Neue Ausbildungsberufe } \\
\text { (Seit Mitte 1997) }\end{array}$ & \multicolumn{2}{l}{ Insgesamt } & \multicolumn{2}{c}{ Frauenanteil } \\
& 2000 & $\begin{array}{l}\text { Zuwachs } \\
2000\end{array}$ & 2000 & 1999 & 1998 & 1997 \\
\hline $\begin{array}{l}\text { Informations- und Telekommu- } \\
\text { nikationselektroniker/in }\end{array}$ & 3375 & +558 & $4 \%$ & $4 \%$ & $4 \%$ & $5 \%$ \\
$\begin{array}{l}\text { Fachinformatiker/in } \\
\begin{array}{l}\text { Informations- und Telekommu- } \\
\text { nikationskaufmann/-kauffrau }\end{array}\end{array}$ & 2953 & +869 & $30 \%$ & $28 \%$ & $26 \%$ & $26 \%$ \\
Informatikkaufmann/-kauffrau & 2494 & +563 & $22 \%$ & $23 \%$ & $23 \%$ & $24 \%$ \\
\hline Summen & 18250 & 5253 & $14 \%$ & $14 \%$ & $13,6 \%$ & $13,8 \%$ \\
\hline
\end{tabular}

Quelle: Deutscher Industrie- und Handelstag (DIHT), 2000.

Als Zwischenfazit lässt sich festhalten, dass die berufliche Ausbildungssituation mit Ausnahme des grafischen Druckbereichs gegenwärtig erst einmal gegen eine künftig erhöhte weibliche Partizipation in den Neuen Medien spricht. Auch die erwähnten geringen Frauenanteile in den technisch und naturwissenschaftlich orientierten Studiengängen lassen überdurchschnittlich erfolgreiche Erwerbsbiografien in den männerdominierten Tätigkeitsbereichen der technischen Produktion von Medienprodukten (Webdesign, Systemadministration, Softwareentwicklung) nur in Ausnahmefällen wahrscheinlich werden.

Ein ergiebiges weibliches Rekrutierungspotenzial für Berufstätigkeiten in der Neuen Medienbranche könnten dagegen die wirtschafts- (rund 40\% Frauenanteil) und kommunikationswissenschaftlichen (knapp unter 60\%) Studiengänge bieten. Damit fehlen Frauen zwar die technischen Kompetenzen, sie scheinen aber gut qualifiziert für die Inhalteproduktion, für Beratungs- und Vermittlungsfunktionen und auch für Führungsund Leitungstätigkeiten. Diese beruflichen Chancen und Potenziale, die für Frauen aufgrund ihrer Qualifikation im Bereich der Neuen Medien gegeben sind, sind im Folgenden auf ihre Realisierungsaussichten zu diskutieren.

\section{Perspektiven von Frauen in Neuen Medienberufen - Eine Diskussion}

Mit dem Übergang zu einer Informationsgesellschaft oder Wissensgesellschaft oder sonst wie bezeichneten "New Society“ wird vielfach auch das Ende des so genannten Normalarbeitsverbältnisses prophezeit: Das dauerhafte, insbesondere unterbrechungslose, perspektivisch sogar lebenslange, qualifizierte Vollzeitarbeitsverhältnis in einem größeren Betrieb (Raasch, 2000: 25) erodiert; dabei werden die Erwerbstätigen künftig nicht nur sequenziell Betriebe und Tätigkeiten, u. U. sogar den Beruf wechseln, sondern auch parallel verschiedene "Jobs“ nebeneinander haben. Die Biografie wird zum Patchwork und Flexibilität hinsichtlich der Erwerbsarbeitsmenge, der -arbeitszeit, des -arbeitsorts und der Arbeitsinhalte und -anforderungen zur Schlüsselqualifikation. Da sich 
Tabelle 2: Zusammenfassung: Ausbildungssituation in IT-und Medienberufen

\section{Schüler}

- Hauptschulen $56 \%$

- Realschulen $49 \%$

- Gymnasien $46 \%$

Duale Ausbildung IT-Berufe

- 40.000 Ausbildungsverträge in ITund Medienberufen

Studienanfänger 2000/2001

- Männer 51\% (absolut: 132.153)

Studienanfänger speziell 1999/2000

- Ingenieurwissenschaften: $78 \%$ (abs. 38.336)

- Maschinenbau: 83\% (abs. 15.033)

- Elektrotechnik 92\% (abs. 10.678)

- Informatik 83\% (abs. 15.726)

- Wirtschaftswissenschaften 53\% (abs. 27.006)

- Medienkunde/Kommunikations-, Informationswissenschaft 36\% (abs. 442)

Studierende speziell 1999/2000: Männer

- Ingenieurwissenschaften: $81 \%$ (abs. 324.430)

- Maschinenbau: 87\% (abs. 84.462)

- Elektrotechnik 95\% (abs. 57.450)

- Informatik 86\% (abs. 73.200)

- Wirtschaftswissenschaften $60 \%$ (abs. 155.543)

- Medienkunde/Kommunikations-, Informationswissenschaft $42 \%$ (abs. 3.565)

\section{Schülerinnen}

- Hauptschulen 44\%

- Realschulen 51\%

- Gymnasien 54\%

Anteil Frauen

- in IT-Berufen: $14 \%$

Studienanfängerinnen 2000/2001

- Frauen 49\% (absolut: 126.501)

Studienanfängerinnen speziell 1999/2000

- Ingenieurwissenschaften: $22 \%$ (abs. 10.689)

- Maschinenbau 17\% (abs. 3167)

- Elektrotechnik 8\% (abs. 1.004)

- Informatik 17\% (abs. 3.273)

- Wirtschaftswissenschaften $47 \%$ (abs. 23.897)

- Medienkunde/Kommunikations-, Informationswissenschaft 64\% (abs. 786)

Studierende speziell 1999/2000: Frauen

- Ingenieurwissenschaften: 19\% (abs. 58.052)

- Maschinenbau: 13\% (abs. 12.758)

- Elektrotechnik 5\% (abs. 3.184)

- Informatik 14\% (abs. 11.920)

- Wirtschaftswissenschaften $40 \%$ (abs. 102.024)

- Medienkunde/Kommunikations-, Informationswissenschaft 58\% (abs. 4.850)

Quelle: in Anlehnung an Kompetenzzentrum Frauen in Informationsgesellschaft und Technologie, ergänzt durch Angaben des Statistischen Bundesamtes.

damit die Norm der modernen Erwerbsarbeit Arbeitsverhältnissen angleicht, wie sie für Frauen bereits seit langem typisch sind, wird in diesem Zusammenhang auch immer wieder von der „Feminisierung der Erwerbsarbeit“ gesprochen (Tischer: 1999, 954).

Auch wenn Frauen schon in der Vergangenheit regelmäßig arbeitspolitische Vorreiterinnen waren (Döbler: 1998, 63ff.) und selbst eine mögliche Feminisierung der Erwerbsarbeit noch lange nicht bedeuten muss, dass Frauen davon auch nachhaltig profitieren werden, steht gleichwohl außer Frage, dass sich Erwerbsarbeit mit dem Einsatz neuer Informationstechnologien wandelt und weiter wandeln wird: Insbesondere der zeitlich und räumlich gleichsam unbegrenzte Austausch von Informationen bei gleich- 
zeitig schneller und kostengünstiger Produktion, Verarbeitung und ebensolchem Transport von großen Informationsmengen vermag die zeitliche und räumliche Fixierung von Arbeitnehmern aufzulösen.

\subsection{Quantitative Beschäftigungsentwicklung}

Eine Folge dieser Erosion des Normalarbeitsverhältnisses soll, zunächst rein quantitativ betrachtet, eine dramatische Verschiebung innerhalb des Dienstleistungsbereichs sein: So wird davon ausgegangen, dass primäre Dienstleistungen von den sekundären Dienstleistungen ${ }^{7}$ quantitativ abgelöst werden. Die durch Rationalisierung ausgelösten absoluten und relativen Beschäftigungsabnahmen werden dabei vor allem gering und unqualifizierte Arbeitskräfte betreffen, während höher- und hoch qualifizierte Dienstleistungsarbeitnehmer/innen eher zu den Gewinnern/innen einer solchen Entwicklung zählen (Tischer, 1999: 950ff.). Die Ausgangsbasis für Frauen stellt sich hier eher ungünstig dar: So waren Frauen ohne formalen beruflichen Abschluss schon in der Vergangenheit überdurchschnittlich von Stellenabbau betroffen, womit sich das Arbeitsplatzrisiko für nicht bzw. gering qualifizierte Frauen mit dieser Entwicklung weiter verstärken wird ${ }^{8}$ Vor allem arbeiten auch viele der formal gut bis sehr gut ausgebildeten und qualifizierten Frauen - u.a. weil sie nicht qualifikationsadäquat eingesetzt werden - in den primären Dienstleistungen oder in hochgradig arbeitsteiligen und standardisierten Arbeitsfeldern ${ }^{9}$, die nun einem verstärktem Rationalisierungsdruck unterliegen werden (Fuzinski et. al., 1997: 218).

Bezogen auf Beschäftigungsperspektiven in den Neuen Medien ist insbesondere das nach wie vor sehr enge Spektrum in der weiblichen Ausbildung als problematisch zu bewerten. Diese immer noch starke geschlechtsspezifische berufliche Differenzierung führt dazu, dass zumindest in den technisch ausgelegten Neuen Medienberufen die relevanten IuK-technologischen Kenntnisse fehlen. Auch wenn im Kontext der Green CardDiskussion und des Mangels an Fachkräften eine technische Ausbildung von Frauen ermutigt und etwa durch reine (geplante) IT-Frauenstudiengänge auch gefördert werden sollen - das Studium wird oftmals als „Königsweg“ in Multimediajobs genannt ${ }^{10}$-, ist für die nahe, aber auch weitere Zukunft eine „Eroberung“ technisch ausgerichteter Bereiche in den Neuen Medien eher unwahrscheinlich. Ohne einen historischen Determinismus zu unterstellen, könnte unter Rückgriff auf historische Parallelen eine Ausbildung hier für Frauen sogar mittelfristig eher eine Sackgasse darstellen; schon öfters und gerade bei Engpässen auf dem Arbeitsmarkt wurden Frauen nämlich auch für klassische Männerbereiche akquiriert, um dann doch in qualifikationsunterwertigen Tätigkeiten „hängen“ zu bleiben oder aber in konjunkturellen und strukturellen Krisensituationen als Erste entlassen zu werden - kurz: von den Männern wieder verdrängt zu werden. An zahlreichen Beispielen aus der Vergangenheit, u. a. auch an dem alten Medienberuf „Pu-

7 Dazu zählen z. B. Managen, Forschen, Entwickeln, Planen oder Publizieren und Informieren.

8 Nach wie vor existiert ein bestimmter Prozentsatz der erwerbstätigen Frauen, die über keine berufliche Ausbildung verfügt: für die alten Länder beträgt dieser z. B. 18\% (Bundesalstalt für Arbeit, 2000: 396).

9 Dazu zählen Schreibdienste, die Datenerfassung und die routinisierten Sachbearbeitungstätigkeiten im Büro.

10 Laut dem Institut der deutschen Wirtschaft wird in den Kernberufen z. B. bei Projektleitern/innen zu $81 \%$, bei Programmierern/innen zu 79\%, bei Conceptionern/innen zu $65 \%$ und bei Screendesignern/innen zu 63\% ein akademischer Abschluss bevorzugt (Tischer, 1998: 47). 
blic Relations“, lässt sich zeigen, dass mit dem Eindringen von Frauen in einen Berufsbereich dieser in der Regel eine tendenzielle Abwertung mit negativen Auswirkungen nicht nur auf das Lohnniveau in diesem Bereich erfährt (Dees/Döbler, 2000: 111ff.). Gerade auch in der Folge des so genannten "Affirmative Action Programs“, das in den USA Gleichstellungsmaßnahmen forderte, zeigte sich mit dem Eindringen von Frauen in vormals typische Männerberufe relativ häufig eine fast durchgängige Substitution der am unteren Ende der Hierarchie befindlichen Männer durch die Frauen, während die Männer zumeist in qualifiziertere Positionen aufrückten ${ }^{11}$ (Ostner, 1983: 138f.).

Besser werden die Möglichkeiten von Frauen in Ausbildungsberufen und Studiengängen beurteilt, in denen kaufmännische, journalistische oder gestalterische Aspekte im Vordergrund stehen; besser nicht nur wegen der hohen Frauenanteile in diesen Bereichen, sondern weil gerade für diese nicht-technischen Neuen Medienbereiche davon ausgegangen wird, dass sich auch neue Bedingungen und Strukturen des Arbeitens herausbilden werden, die Frauen begünstigen bzw. der weiblichen Erwerbsbiografie besser entsprechen sollen. Doch auch diese, oftmals unter dem Oberbegriff Flexibilisierung subsumierten Tendenzen und prognostizierten Entwicklungen sind bei einer differenzierteren Analyse eher kritisch, in jedem Fall aber ambivalent zu bewerten.

\subsection{Qualitative Beschäftigungsentwicklung}

Als hervorragende Punkte werden regelmäßig die durch die neuen Technologien bedingten zeitlichen und räumlichen Flexibilisierungspotenziale genannt, die insbesondere für Frauen die Erwerbschancen nachhaltig verbessern würden, indem berufliche und familiale Lebensbereiche konfliktfreier verknüpft werden könnten. So gelten etwa heute schon „Normalarbeitszeiten“mit acht Stunden Arbeit pro Tag und das an fünf Tagen in der Woche als Auslaufmodell (Wilke, 1999: 89). Nun erfolgt die zeitliche Flexibilisierung der Erwerbsarbeit aber in erster Linie entlang betrieblicher Anforderungen; nicht primär die Zeitbedürfnisse der Beschäftigten, sondern die Schwankungen auf den Beschaffungs- und Absatzmärkten oder der Wunsch nach Verlängerung der Betriebsnutzungszeiten sind die Bestimmungsgründe für die Flexibilisierung von Arbeitszeiten. Geht man davon aus, dass auch künftig die Frauen sich stärker für familiale Belange verantwortlich fühlen bzw. verantwortlich gemacht werden, ist für eine grundlegende Verbesserung weiblicher Lebensentwürfe jedoch vor allem die autonome Zeitsouveränität von Bedeutung (Winker, 1998: 18). Und selbst wenn Arbeitszeiten überwiegend im Einverständnis mit den Arbeitnehmern/innen und damit nicht gegen deren Zeitrahmen gestaltet werden, bleiben die individuellen Handlungsspielräume dabei begrenzt und sollten in den Neuen Medien nicht überschätzt werden. Gerade im Bereich der Film-, Fernseh- und Hörfunkproduktion ist die Zeitsouveränität der Beschäftigten ohnehin stark eingeschränkt, da die Produktionsbedingungen dieser Branche dies oft nicht zulassen. Ebenso belegen Studien zu den Arbeitsbedingungen im Multimediabereich, dass die hier üblichen starken Schwankungen im Arbeitsvolumen zwar hoch flexibel aufgefangen werden, aber alles andere als frauen- und familienfreundlich organisiert sind: So kommt es in Abhängigkeit von Projektarbeiten und dem damit verbundenen Termindruck in den so genannten Hochphasen oft zu erheblichen Überstunden im Büro, zu Arbeit an

11 „Masculine gender identity is defined as that which is not feminine, so men are more instrumental than women in establishing that difference. ... Women do not seem threatened when men enter a predominantly female occupation. ..." (Williams, 1989: S. 135). 
den Wochenenden und es wird selbstverständlich, Arbeit mit nach Hause zu nehmen. (Liesering, 1999: 994). Darüber hinaus schränkt die Innovationsgeschwindigkeit der Branche die Anwendbarkeit von solchen Arbeitszeitmodellen stark ein, die mit einer Unterbrechung der Erwerbstätigkeit (z. B. Erziehungsurlaub) verbunden sind. Schon eine vergleichsweise kurze Unterbrechung von ein paar Monaten bis zu einem Jahr ${ }^{12}$ geht mit einem dramatischen Qualifikationsverlust und Karriereknick einher, so dass hier der Konflikt zwischen Familie und Beruf eher noch verschärft wird.

Anders als die zeitliche Flexibilisierung steckt die räumliche Flexibilisierung in Deutschland entgegen den zeitweise euphorischen Prognosen und im Vergleich mit anderen europäischen Ländern oder den USA noch in den Kinderschuhen. ${ }^{13}$ Gleichwohl scheinen etliche der Tätigkeiten in den Neuen Medien - wie auch schon in den alten Medien -, etwa ein Großteil der journalistisch oder gestalterisch angelegten Tätigkeiten, daneben auch Programmierungs- und Entwicklungstätigkeiten für eine räumliche Flexibilisierung und insbesondere für die so genannte Tele(heim)arbeit prädestiniert. Da Tele(heim)arbeit gerade unter dem Aspekt einer besseren Vereinbarkeit von Familie und Beruf diskutiert und propagiert wird, scheint diese Art der räumlichen Flexibilisierung den Frauen entgegenzukommen und es sind auch die Frauen unter 30 Jahren, die ein deutlich überproportionales Interesse an Tele(heim)arbeit bekunden (Wilke, 1999: 121). Bislang sind drei Viertel der Tele(heim)arbeiter/innen in Deutschland und in Europa aber zunächst erst einmal männlich (Schwarze, 2000). Zweifelhaft scheint jedoch, ob eine Erhöhung des Frauenanteils in Tele(heim)arbeitsplätzen überhaupt wünschenswert ist: Die unmittelbare räumliche Nähe zu Haus und Familie wird mit Isolierung vom sozialen Kontext der Arbeit, potenzieller Vereinsamung und Lösung sozialer Bindungen zu den Kollegen/innen erkauft; zudem besteht die Gefahr der Selbstausbeutung, da die „rund um die Uhr Verfügbarkeit“ zu einer Aufhebung der traditionellen Trennung von Nicht-Arbeit und Arbeit führt - die Probleme der Doppelbelastung könnten hierdurch eher noch verschärft werden. ${ }^{14}$ Als gewichtiges Gegenargument müssen zudem die verminderten Karrierechancen gegenüber Nicht-Telearbeitern/innen in Betracht gezogen werden (Wilke, 1999: 126), die gerade einer Erhöhung des Frauenanteils in Führungsfunktionen eher abträglich wären. Vor allem aber könnte vermehrte Tele(heim)arbeit von Frauen zu einer Zementierung, wenn nicht sogar zu einer Renaissance traditioneller geschlechtsspezifischer Rollenzuschreibungen führen und damit manche Emanzipationserfolge der Vergangenheit zunichte machen.

Eine alternative Organisation der Tele(heim)arbeit, etwa in Form der alternierenden Telearbeit, die in der Praxis gern angewandt wird, um die Nachteile reiner Tele(heim)arbeit zu mindern, mag auch für Frauen eine attraktive Lösung darstellen, berufliche und außerberufliche Lebensbereiche in einen harmonischeren Einklang zu bringen; allerdings steht auch hier zu befürchten, dass - zumindest wenn mehr Frauen als Männer so arbeiten sollten - gesellschaftliche Etikettierungsprozesse zu einer Abwertung dieser Form der beruflichen Erwerbsarbeit führen.

12 In der Regel wird hier ein Internetjahr mit sieben ,normalen' Jahren gleichgesetzt.

13 Für das Jahr 1999 wird die Zahl der Tele-Arbeitsplätze bei ca. 33 Millionen Erwerbstätigen auf rund eine Million geschätzt.

14 So gaben z. B. in einer Untersuchung des BMA nur Frauen als Grund für die Telearbeit die Erziehung von Kindern an, bei Männern dagegen spielte dieses Motiv keine Rolle (Kühlwetter, 1999: 1008). 
Auch eine weitere prognostizierte Entwicklung, nämlich dass die Erwerbsarbeitsmenge künftig verstärkt in Abhängigkeit betrieblicher Erfordernisse flexibel auf einzelne Beschäftigte verteilt wird, dürfte den Frauen nicht zum Vorteil gereichen. Eine solche Flexibilisierung geht mit einer verschärften Polarisierung des Arbeitsmarktes einher: auf der einen Seite überbeschäftigte Stammbelegschaften mit gesichertem Erwerbseinkommen, aber auch Arbeitszeiten von wöchentlich bis zu 60 Stunden, auf der anderen Seite eine flexibel einsetzbare Randbelegschaft, niedrig entlohnt, in Teilzeitarbeits- oder zeitlich befristeten Arbeitsverhältnissen, Leiharbeitnehmer/innen und Scheinselbstständige (Winker, 1998: 16).

Frauen befinden sich schon heute mehr als Männer in atypischen Beschäftigungsverhältnissen ${ }^{15}$, mit einer weiteren Polarisierung wächst die Gefahr einer weiteren Abdrängung von Frauen in diese sozial nicht oder nur wenig gesicherten Arbeitsverhältnisse. Eine Polarisierung in eine männliche Stammbelegschaft mit gesicherten, gut bezahlten Tätigkeiten und eine weibliche Randbelegschaft in sozial unsicheren und schlecht bezahlten Jobs ist zwar eher untypisch für die gegenwärtige Medienwirtschaft - hier ist etwa der Anteil an geringfügig Beschäftigten bislang vergleichsweise gering - einer Experten/innenbefragung zufolge wird aber auch die künftige Medienwirtschaft von dieser Entwicklung betroffen sein (Fuzinski et. al., 1997: 95). Schon jetzt greift die Medienbranche, alte und neue Medien (von der Werbewirtschaft über die Verlagsbranche und die audiovisuellen Medien bis zur neuen Multimediabranche), in großem Umfang auf freie Mitarbeiter/innen ${ }^{16}$ zurück, und speziell in der Multimediawirtschaft ist der Anteil freier Mitarbeiter/innen sehr hoch. Daneben spielen externe Dienstleistungen für das gesamte Spektrum an technischen und gestalterischen Aufgaben eines Medienproduzenten eine bedeutende Rolle. Unternehmensbefragungen in der Branche zeigen, dass die Funktionsaufteilung in den Multimedia-Units der Verlage meist identisch sind: Feste Mitarbeiter/innen gibt es überwiegend in den Bereichen Produktmanagement und Marketing sowie teilweise im technischen Bereich (Programmierer/innen und Netzwerkspezialisten/innen $)^{17}$, während das Gros der Arbeiten für Multimediadesign und Programmierung extern von Selbstständigen erfolgt (Benkert/Michel, 1999: 24f.). Auch wenn aufgrund des fehlenden Datenmaterials derzeit noch nicht abgeschätzt werden kann, inwieweit Frauen in den Neuen Medien hiervon tatsächlich verstärkt betroffen sein werden, ist wiederum unter Rückgriff auf historische Analogien davon auszugehen.

Weisen diese Flexibilisierungspotenziale somit sogar zu einem guten Teil eher frauenund familienfeindliche Tendenzen auf, so bleibt noch das gerne vorgebrachte Argument, dass innerhalb der Neuen Medien eine gewisse Entformalisierung und Enthierarchisierung stattfindet: Das Aufbrechen traditioneller („patriarchalischer") Organisationsstrukturen mit flacheren Hierarchien, mit stärker inhaltlich und projektbezogenen Arbeiten in dezentral organisierten Teams (Tischer, 1998: 42f.) komme dem weiblichen Arbeitsvermögen entgegen und würde damit Frauen neue Möglichkeiten und Perspektiven geben. Denn mit den Strukturen würden sich auch die Anforderungen an Mitarbeiter/innen wandeln: Reines Fachwissen verliere, Methoden- (z. B. Planung- und Or-

151995 war nicht einmal jede zweite erwerbstätige Frau in den alten Länder in einem „Normalarbeitsverhältnis“, d. h. in einer unbefristeten abhängigen Vollzeitbeschäftigung, bei Männern dagegen sind dies zwei Drittel (Tischer, 1999: 954).

16 Vgl. zur Definition von „freier Mitarbeit“ z. B. Böhm/Volkert, 1998: 4ff.

17 Hier spielt der Zugang zu Codes bei der Programmierung von Serienprodukten die entscheidende Rolle. 
ganisationsvermögen) und Sozialkompetenz (Teamfähigkeit, Kommunikationsfähigkeit oder Kontaktfähigkeit) gewannen dagegen an Gewicht - und damit gerade solche Kompetenzen, die traditionell als „weiblich“ definiert gelten, d. h. Frauen besonders zugeschrieben werden und damit Frauen für die neuen Anforderungen besser qualifizierten. So wird etwa für Online-Redaktionen davon ausgegangen, dass sich die eher traditionellen Führungsstrukturen im „herkömmlichen“ Verlagsbereichs nicht reproduzieren, sondern „modernere“ Strukturen mit veränderten Qualifikationsanforderungen herausbilden, die besonders in der Umbruchphase Frauen vermehrte Chancen eröffnen (Fuzinski et al., 1997: 207). Damit nicht genug: Als weitere Kernkompetenzen werden für die Multimedia- und IuK-Branche Navigationskompetenz, Recherchekompetenz, logisches und vernetztes Denken, Experimentierwille und Kreativität, Beurteilungs-, Auswahl- und Einsatzkompetenz sowie Weiterbildungsbereitschaft (Fuzinski et. al., 1997: 84) genannt. Auch in den Kernberufen der Informationstechnologie ist also eine stärker interdisziplinäre Ausbildung gefragt, die nicht nur ausschließlich Spezialkenntnisse in Technik erfordert, womit Frauen auch in diesen Feldern mit Mischqualifikation profitieren könn(t)en. Kühlwetter sieht besonders gute Chancen für Frauen an der Schnittstelle Mensch/Technik, z. B. im Vertrieb, Marketing und in der Kundenberatung, da sie sich stärker auf die Bedürfnisse der Kunden einlassen. Weitere aussichtsreiche Betätigungsbereiche sieht sie im „content“-Bereich, wo hauptsächlich konzeptionelle, dramaturgische, gestalterische und pädagogische Fähigkeiten gefordert sind und Frauen sich aktiv in die Technikgestaltung einbringen könnten (Kühlwetter, 1999: 1000).

Doch auch diese Prognosen sind kritisch zu differenzieren und zu relativieren. Wetterer (1992: 22ff.) etwa führt aus, dass sich Frauen benachteiligende Strukturen im Erwerbsleben weitgehend unabhängig von deren Ausbildung und Qualifikation finden und postuliert eine Form institutionalisierter Ungleichheit zwischen den Geschlechtern, die auch bei gleicher Qualifikation fortbesteht: Entgegen herkömmlichen, auch im Rahmen der neoklassischen Angebotstheorien getroffenen Annahmen kommt der Qualifikation nicht die Schlüsselfunktion bei der Verteilung beruflicher Chancen zu. Vielmehr dominiert das Geschlecht über die Qualifikation, es findet eine geschlechtsspezifische Hierarchisierung statt. Eine Ausbildung von Hierarchien zwischen den Geschlechtern zeigt sich historisch dabei nicht nur innerhalb eines bestimmten Berufs, sondern gleich über ganze Berufsfelder hinweg. Selbst in überwiegend den Frauen überlassenen Berufen dominiert die Minderheit der Männer sowohl in den oberen als auch in den bedeutenderen Positionen (Döbler, 1998: 66ff.). Und findet in einem Berufsbereich eine Feminisierung statt, so ist dies oftmals mit einer generellen Abwertung von Tätigkeitsanforderungen und Qualifikationsmerkmalen dieser Arbeitsbereiche verbunden: Verkürzt lässt sich sagen, dass Qualifikationen, die überwiegend bei Frauen anzutreffen sind, eher gering geschätzt werden, was sich u. a. in einer schlechteren Entlohnung und in einer niedrigeren hierarchischen Positionierung niederschlägt. Selbst wenn man nicht in Abrede stellt, dass Frauen tatsächlich gehäuft über spezifische und auch nachgefragte Kompetenzen verfügen - in jedem Fall ist diese Aussage erst noch erheblich zu präzisieren - sind auch die vielfältigen Hoffnungen, die hinsichtlich der beruflichen Perspektiven von Frauen in den Neuen Medien formuliert werden, doch mit einer gehörigen Portion Skepsis zu belegen. Auch in den Neuen Medienberufen könnte sich ein aus anderen Berufen gewohntes Strukturierungsmuster bestätigen, nach welchem die Frauen, obwohl mehrheitlich in bestimmten Bereichen vertreten, obwohl mit relevanten Kompetenzen und Qualifikationen gut ausgestattet, in den hierarchisch niederen und finanziell unattraktiveren Positionen verbleiben, während der zahlenmäßig 
unterlegenen Gruppe der Männer trotz Defiziten in der Sozial- und Methodenkompetenz der Aufstieg in der organisatorischen und in der Entgelt-Hierarchie gelingt.

\section{Ausblick}

Die Funktion von Qualifikationen und Kompetenzen kann als relativ eingestuft werden - insofern sind auch gewisse formale Ausbildungsvorteile in kaufmännischen, journalistischen oder kreativen Bereichen oder auch die gerne pauschal unterstellte höhere Sozial- und Methodenkompetenz von Frauen zwar wichtige Kriterien für das Entree in die Neue Medienbranche, sollten aber nicht überschätzt werden. Gelingt es aber Frauen tatsächlich, in nennenswerter Anzahl in spezifische Positionen, u. U. sogar in Führungspositionen in den Neuen Medien vorzustoßen, so ist damit zu rechnen, dass diese feminisierten Bereiche dann sowohl unternehmensintern als auch -extern eine gewisse Abwertung erfahren werden. Denkbar ist auch, dass bestimmte Bereiche ganz den Frauen überlassen, gleichzeitig diese aber mehr oder minder straff in organisatorische Strukturen, die etwa auf den weiterhin männlich beherrschten Technikbereich ausgelegt sind, eingegliedert werden. Zweifellos bieten die Neuen Medien interessante neuartige Betätigungsfelder und Berufskarrieren für Frauen - auch durch die vielfältigen, hier nicht diskutierten Möglichkeiten der Unternehmensgründung -, aber als gleichsam emanzipatorischer Hort, in denen gesellschaftlich verfasste geschlechtsspezifische berufliche Segregationen überwunden werden, scheinen sie zum gegenwärtigen Zeitpunkt doch bei weitem überschätzt.

\section{Literatur}

Benkert, W./Michel, L. P. (1999): Neue Selbständige in der Medienbranche. Ein Modellfall für den Dienstleistungssektor? Stuttgart (Arbeitsbericht Nr. 138 der Akademie für Technikfolgenabschätzung).

Böhm, D./Volkert, B. (1998): Freie Mitarbeit im Multimedia-Markt. Stuttgart (Arbeitsbericht Nr. 127 der Akademie für Technikfolgenabschätzung).

Bund-Länder-Kommission für Bildungsplanung und Forschungsförderung (BLK) (2000): Verbesserung der Chancen von Frauen in Ausbildung und Beruf. Ausbildungs- und Studienwahlverhalten von Frauen. Bonn (Materialien zur Bildungsplanung und zur Forschungsförderung, Heft 80).

Bundesanstalt für Arbeit (2000): Arbeitsmarkt für Frauen. Aktuelle Entwicklungen und Tendenzen im Überblick. Sonderausdruck aus den Amtlichen Nachrichten der Bundesanstalt für Arbeit (ANBA) Nr. 4/2000.

Bundesinstitut für Berufsbildung (2001): Die neuen IT-Berufe. In: http://www.bibb.de/forum/itberufe/erfolgsstory.htm (Abruf 8.5.2001).

Dees, M./Döbler, T. (2000): Public Relations als Aufgabe für Manager? Rollenverständnis, Professionalisierung, Feminisierung - Eine empirische Untersuchung, Stuttgart.

Döbler, T. (1998): Frauen als Unternehmerinnen. Erfolgspotentiale weiblicher Selbständiger. Wiesbaden.

Engelbrech, G./Jungkunst, M. (1999): Die Zukunft der Frauenbeschäftigung. Veränderung der Arbeitslandschaft nach Tätigkeiten und Qualifikationsebenen zwischen 1995 und 2010. IABWerkstattbericht Nr. 20/1999, Nürnberg.

Engelbrech, G./Reinberg, A. (1998): Erwerbsorientierung und Beschäftigungsmöglichkeiten von Frauen in den neunziger Jahren. Wirtschaftliche Umstrukturierung und frauentypische Arbeitsmarktrisiken in Ost- und Westdeutschland. In: Gesellschaft für Informationstechnologie und Pädagogik am IMBSE (Hrsg.): Beschäftigungsrisiko Erziehungsurlaub. Die Bedeutung des „Erziehungsurlaubs“ für die Entwicklung der Frauenerwerbstätigkeit. Opladen, S. 39-91.

Fuzinski, A. et al. (1997): Herausforderung Informationsgesellschaft: Auswirkungen neuer Infor- 
mations- und Kommunikationstechnologien auf die Beschäftigungssituation von Frauen. Eine Studie im Auftrag des Ministeriums für die Gleichstellung von Frau und Mann des Landes Nordrhein-Westfalen. Düsseldorf.

Initiative D 21 (2001): Die Entwicklung des Arbeitsmarktes und der Hochschulplätze für IT-Fachkräfte in Deutschland. Zwischenergebnis der Arbeitsgruppe „Bildung und Qualifikation“. Frankfurt/ Stuttgart.

Klatt, R./Richter-Witzgall, G. (2000): Frauen in Zukunftsberufen - Wege zu einer wirtschaftsnahen Entwicklung der Chancengleichheit von Frauen in der Ausbildung. Expertise. Im Auftrag des Ministeriums für Frauen, Jugend, Familie und Gesundheit des Landes Nordrhein-Westfalen. Sozialforschungsstelle Dortmund.

Kluge, D. (2001): Ende des Job Booms. Die Zeit der Quereinsteiger ist vorbei. In: Spiegel Online vom 16. Mai 2001. In: http://www.spiegel.de/netzwelt/ebusiness.html (Abruf 16.5.2001).

Kühlwetter, K (1999): Multimedia - Chance für Frauen. Qualifizierungsmöglichkeiten für berufliche Tätigkeiten mit multimedialen Techniken und Systemen. In: Bundesanstalt für Arbeit: Frauen in der Informationsgesellschaft. Informationen für die Beratungs- und Vermittlungsdienste der Bundesanstalt für Arbeit ibv Nr. 13/1999, Nürnberg, S. 999 - 1004.

Liesering, S. (1999): Chancen von Frauen im Multimediabereich. Ergebnisse einer empirischen Untersuchung zur Beschäftigungssituation von Multimediaspezialistinnen. In: Bundesanstalt für Arbeit: Frauen in der Informationsgesellschaft. Informationen für die Beratungs- und Vermittlungsdienste der Bundesanstalt für Arbeit ibv Nr. 13/1999, Nürnberg, S. 985 - 998.

Ostner, I. (1983): Berufsform und berufliche Sozialisation von Frauen. In: Bolte, K. M./Treutner, E. (Hrsg.): Subjektorientierte Arbeits- und Berufssoziologie. Frankfurt/M., S. $110-140$.

Raasch, S. (2000): Erosion im (männlichen) Normalarbeitsverhältnis - Chance für eine Neuverteilung von bezahlter Arbeit? In: Konrad-Adenauer-Stiftung (Hrsg.): Die Abendröte der Industriegesellschaft - Chance für Frauen? Sankt Augustin.

Schwarze, B. (2001): Arbeit, Bildung und Privates im Zeichen des Internets - In Zukunft alle IT? Kompetenzzentrum. Frauen in Informationsgesellschaft und Technologie. In: http://www. kompetenz.de/seiten/download.html (Abruf 8.5.2001).

Tischer, U. (1998): Neue Beschäftigungsfelder und weibliche Qualifikationspotentiale. In: Winker, G./Oechtering, V. (Hrsg.): Computernetze - Frauenplätze. Frauen in der Informationsgesellschaft. Opladen, S. $33-56$.

Tischer, U. (1999): Arbeit im Wandel. Thesen zu Chancen und Risiken von Frauen in der Informationsgesellschaft. In: Bundesanstalt für Arbeit: Frauen in der Informationsgesellschaft. Informationen für die Beratungs- und Vermittlungsdienste der Bundesanstalt für Arbeit ibv Nr. 13/1999. Nürnberg, S. 949 - 970.

Wetterer A. (1992): Hierarchie und Differenz im Geschlechterverhältnis. In: Wetterer A. (Hrsg.): Profession und Geschlecht - Über die Marginalität von Frauen in hochqualifizierten Berufen. Frankfurt/M., New York, S. $13-40$.

Winker, G. (1998): Virtuelle Unordnung im Geschlechterverhältnis. Umverteilung von Arbeit als Chance? In: Winker, G./Oechtering, V. (Hrsg.): Computernetze - Frauenplätze. Frauen in der Informationsgesellschaft. Opladen, S. $13-32$.

Wilke, G. (1999): Die Zukunft unserer Arbeit. Frankfurt/Main.

Williams, C. (1989): Gender Differences at Work. Women and Men in nontraditional Occupations. Berkeley. 